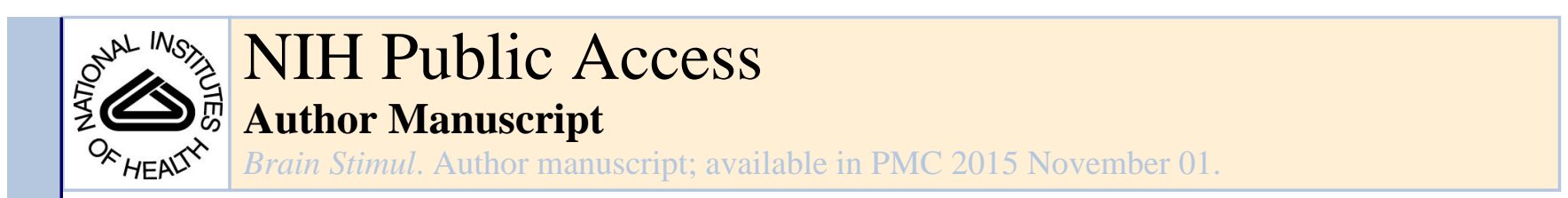

Published in final edited form as:

Brain Stimul. 2014 ; 7(6): 878-889. doi:10.1016/j.brs.2014.07.033.

\title{
Endogenous Cortical Oscillations Constrain Neuromodulation by Weak Electric Fields
}

\author{
Stephen L. Schmidt ${ }^{1,5}$, Apoorva K. lyengar ${ }^{2}$, A. Alban Foulser ${ }^{2}$, Michael R. Boyle ${ }^{1,5}$, and \\ Flavio Fröhlich ${ }^{1,3,4,5,6}$ \\ ${ }^{1}$ Department of Psychiatry, University of North Carolina at Chapel Hill, Chapel Hill NC 27599 \\ 2 Department of Biology, University of North Carolina at Chapel Hill, Chapel Hill NC 27599 \\ ${ }^{3}$ Neurobiology Curriculum, University of North Carolina at Chapel Hill, Chapel Hill NC 27599 \\ ${ }^{4}$ Department of Cell Biology and Physiology, University of North Carolina at Chapel Hill, Chapel \\ Hill NC 27599 \\ ${ }^{5}$ Department of Biomedical Engineering, University of North Carolina at Chapel Hill, Chapel Hill \\ NC 27599
}

${ }^{6}$ Neuroscience Center, University of North Carolina at Chapel Hill, Chapel Hill NC 27599

\section{Abstract}

Background-Transcranial alternating current stimulation (tACS) is a non-invasive brain stimulation modality that may modulate cognition by enhancing endogenous neocortical oscillations with the application of sine-wave electric fields. Yet, the role of endogenous network activity in enabling and shaping the effects of tACS has remained unclear.

Objective-We combined optogenetic stimulation and multichannel slice electrophysiology to elucidate how the effect of weak sine-wave electric field depends on the ongoing cortical oscillatory activity. We hypothesized that the structure of the response to stimulation depended on matching the stimulation frequency to the endogenous cortical oscillation.

Methods-We studied the effect of weak sine-wave electric fields on oscillatory activity in mouse neocortical slices. Optogenetic control of the network activity enabled the generation of in vivo like cortical oscillations for studying the temporal relationship between network activity and sine-wave electric field stimulation.

\footnotetext{
(C) 2014 Elsevier Inc. All rights reserved.

Correspondence should be addressed to: Flavio Frohlich, 115 Mason Farm Rd. NRB 4109F, Chapel Hill, NC. 27599. flavio_frohlich@med.unc.edu.

Publisher's Disclaimer: This is a PDF file of an unedited manuscript that has been accepted for publication. As a service to our customers we are providing this early version of the manuscript. The manuscript will undergo copyediting, typesetting, and review of the resulting proof before it is published in its final citable form. Please note that during the production process errors may be discovered which could affect the content, and all legal disclaimers that apply to the journal pertain.

Authorship Statement: SS and FF designed the experiments; SS, AI, and AF performed the experiments; SS analyzed the data; MB validated the analysis code, and SS and FF wrote the paper.

The authors declare no competing financial interests.

Please note that a minute subset of the data included in this submission was previously presented in abstract form (6 ${ }^{\text {th }}$ IEEE Neuroengineering Meeting 2013 and SfN 2013, both San Diego).
} 
Results-Weak electric fields enhanced endogenous oscillations but failed to induce a frequency shift of the ongoing oscillation for stimulation frequencies that were not matched to the endogenous oscillation. This constraint on the effect of electric field stimulation imposed by endogenous network dynamics was limited to the case of weak electric fields targeting in vivo-like network dynamics. Together, these results suggest that the key mechanism of tACS may be enhancing but not overriding of intrinsic network dynamics.

Conclusion-Our results contribute to understanding the inconsistent tACS results from human studies and propose that stimulation precisely adjusted in frequency to the endogenous oscillations is key to rational design of non-invasive brain stimulation paradigms.

\section{Keywords}

transcranial alternating current stimulation; tACS; optogenetics; electric field; multielectrode array; resonance

\section{Introduction}

Oscillatory activity in neocortex enables temporal organization of information during sensory processing, cognition, and memory $[1,2]$. Perturbing cortical oscillations with brain stimulation has become a key experimental approach to evaluate the functional, frequencyspecific roles of cortical oscillations. Targeted enhancement of cortical oscillations may in the future also serve as a treatment for psychiatric disorders associated with impaired cortical oscillations such as schizophrenia and autism [3]. Suprathreshold excitation of cortical networks with pulsed transcranial magnetic stimulation (TMS) can alter temporal structure of network activity and mediate changes in associated cognitive processes [4, for example 5,6]. Recently, transcranial alternating current stimulation (tACS) has emerged as an alternative non-invasive brain stimulation approach for targeted modulation of cortical oscillations [7]. TACS applies a weak, sine-wave electric field (EF) that targets neocortical networks. Several studies have demonstrated cognitive enhancement by tACS in humans [8-11] and animals [12] and enhancing effects of tACS on neuronal activity in animal models in vitro [13-15] and in vivo [16, 17]. However, very little is known about the interaction dynamics between endogenous cortical oscillations and the small, periodic changes in membrane voltage caused by tACS. In fact, differences in endogenous network activity alter the effects of tACS on macroscopic brain activity measured by electroencephalography (EEG) and may explain the variability of behavioral effects for seemingly very similar stimulation paradigms [18]. In the long term, elucidating the role of endogenous activity in shaping the response to non-invasive brain stimulation is crucial for the development of individualized, adaptive stimulation paradigms that take into account ongoing network activity and adjust stimulation accordingly.

Computational simulations suggest that cortical activity is preferentially enhanced by EFs at the frequency of the endogenous oscillation through network resonance [15, 17]. Network resonance in this contexts is defined as the preference of a network to oscillate in a narrow frequency band in response to periodic stimulation [for review of resonance see 19], in this case centered on the frequency of the endogenous oscillation. Here, we combine optogenetic network activation with applied EF stimulation in vitro to determine how the relationship 
between the network oscillation and stimulation frequency determines the effect of periodic stimulation on cortical oscillations. Using optogenetic stimulation for generating an in vivolike activity structure provided precise and simultaneous control of both the "endogenous" activity [20] and the applied EF stimulation to directly study the interaction dynamics. Specifically, we used optogenetic stimulation of layer 5 pyramidal cells in cortical slices of the Thy1-ChR2 mouse [21] to induce a pronounced slow cortical oscillation $(1 \mathrm{~Hz})$ that we then targeted with applied EF stimulation. Layer 5 pyramidal cells play a central role in lowfrequency cortical oscillations [22] and their optogenetic activation in vivo induces network activity that closely mimics spontaneous slow cortical oscillations [20]. Using this approach, we tested the hypothesis that intrinsically oscillating neocortical networks exhibit network resonance by preferentially responding to frequency-matched sine-wave EF stimulation.

\section{Methods}

All procedures were approved by The University of North Carolina at Chapel Hill Institute of Animal Use and Care and were in compliance with the National Institute of Health guide for care and use of laboratory animals (NIH Publications No. 8023, revised 1978).

\section{Slice Preparation and Data Acquisition}

Thy1-Chr2 mice (Jackson Laboratory, Bar Harbor, ME), deeply anesthetized with Euthasol $(0.5 \mathrm{~mL} / \mathrm{kg}$, Virbac, Fort Worth, TX), were decapitated and their brains extracted and quickly placed in ice-cold sucrose solution (mM: $83.0 \mathrm{NaCl}, 2.5 \mathrm{KCl}, 0.5 \mathrm{CaCl}_{2}, 3.3$ $\mathrm{MgSO}_{4}, 1 \mathrm{NaH}_{2} \mathrm{PO}_{4}, 26.2 \mathrm{NaHCO}_{3}, 22.0$ Dextrose Anhydrose and 72.0 Sucrose) bubbled with carbogen $\left(95 \% \mathrm{O}_{2}, 5 \% \mathrm{CO}_{2}\right) .200 \mu \mathrm{m}$ coronal sections containing $\mathrm{V} 1$ were cut using a vibratome (Leica Microsystems, Wetzlar, Germany) and allowed to recover in incubation solution (mM: $119.0 \mathrm{NaCl}, 2.5 \mathrm{KCl}, 1.0 \mathrm{NaH}_{2} \mathrm{PO}_{4}, 26.2 \mathrm{NaHCO}_{3}, 22.0$ glucose, $2 \mathrm{MgSO}_{4}$ and $2.0 \mathrm{CaCl}_{2}$ ) for at least $1 \mathrm{hr}$ at $34{ }^{\circ} \mathrm{C}$. After recovery, slices were placed on a MEA 2100 (Multichannel Systems, Reutligen, Germany) and perfused with artificial cerebrospinal fluid (aCSF, mM: $119.0 \mathrm{NaCl}, 4.5 \mathrm{KCl}, 1 \mathrm{NaH}_{2} \mathrm{PO}_{4}, 26.2 \mathrm{NaHCO}_{3}, 22.0$ glucose, $1.0 \mathrm{MgSO}_{4}$, and $1.0 \mathrm{CaCl}_{2}$ ), heated to $36^{\circ} \mathrm{C}$, at a rate of greater $4 \mathrm{~mL}$ per minute as in [23]. The calcium and magnesium concentrations in the aCSF were modeled after human CSF concentrations [24]. Data were recorded from 59 electrodes ( $30 \mu \mathrm{m}$ diameter) in a 6 by 10 perforated array with $100 \mu \mathrm{m}$ spacing between electrodes at a rate of $25 \mathrm{kHz}$.

\section{Experimental Design}

Experiments included continuous $1 \mathrm{~Hz}$ optogenetic stimulation by blue light $(460 \mathrm{~nm}, 22$ $\mathrm{mW}$ maximum, approximately $200 \mu \mathrm{W}$ delivered at slice surface) from a LED 460 (Prizmatix, Givat Shmuel, Israel). EF was generated by applying a calibrated current from a stimulus isolator (World Precision Instruments, Sarasota, FL) between two $\mathrm{AgCl}$ wires in the bath. Slices were arranged on the MEA such that pia was in parallel to the stimulation wires and therefore approximately orthogonal to the somato-dendritic axes of the pyramidal cells in the slice preparation. Therefore, the EF was oriented from the wire close to layer 1 of visual cortex to the wire close to the ventral side of the slice such that the positive first half-wave and the negative second half wave caused a somatic membrane depolarization and hyperpolarization, respectively [25, 26]. Experiments consisted of continuous $1 \mathrm{~Hz}$ 
optogenetic stimulation (square-wave, 50\% duty cycle) for 64 trials of EF stimulation. Each trial of EF stimulation consisted of $10 \mathrm{~s}$ of optogenetic stimulation, $10 \mathrm{~s}$ of EF with optogenetic stimulation, followed by an additional $10 \mathrm{~s}$ of optogenetic stimulation. The order of trials was randomized for both stimulation frequency $(0.8,1.0,1.2 \mathrm{or} 2.0 \mathrm{~Hz})$ and stimulation amplitude ( 1 or $2 \mathrm{mV} / \mathrm{mm} \mathrm{EF}$ ). The EF was selected to be of similar amplitude to the estimated EF strength during tACS in humans $[11,27]$ and was below action potential threshold for cells at rest $[14,26]$. For experiments without optogenetic stimulation, the same stimulation paradigm was used however the LED was disconnected from the power supply. In experiments with low intensity optogenetic stimulation, the intensity was decreased to an intensity of $1 / 10^{\text {th }}$ of the full intensity (approximately $20 \mu \mathrm{W}$ at slice surface). For experiments with varied optogenetic stimulation, we first selected the length of each "UP/DOWN state" from a normal distribution (mean $500 \mathrm{~ms}$, standard deviation 50 ms) for a total of 10 oscillation cycles. "UP" states here refer to the periods when the LED was on. Conversely, "DOWN" states refer to the inactive phase of the LED stimulation cycle.

\section{Data Analysis}

Multiunit traces and stimulation data were recorded using MC_Rack (Multichannel Systems, Reutligen, Germany). All data analysis was performed by custom-written Matlab (Mathworks, Natick, MA) scripts. Spikes were extracted at the time-points at which the recorded, high-pass filtered trace for each electrode crossed the threshold of -4 times the standard deviation of the trace with a $1 \mathrm{~ms}$ dead time. Application of the EF would occasionally cause a transient, stereotyped voltage spike on all electrodes at the onset, offset or zero-crossing of EF cycles; therefore artifact removal was performed by finding and removing voltage spikes that occurred on 10 or more electrodes within $1 \mathrm{~ms}$. Following artifact removal, only electrodes in V1 were included for subsequent analysis. Single units (SU) were isolated using methods outlined in [23, 28, 29]. Spike trains were binned with 1 ms resolution for each SU. The FR effect of EF stimulation was calculated as the FR during EF stimulation divided by the FR of the period without EF stimulation immediately preceding stimulation for each electrode for each trial. The activation ratio was calculated as the number of spikes while the LED was on divided by the total number of spikes. Wholeslice spike trains were constructed by summing spike trains across electrodes ( $1 \mathrm{~ms}$ bins). Frequency transforms were calculated by convolution with Morelet wavelets followed by temporal averaging, and subsequent whole-slice spectra were then normalized by dividing by the number of summed SU. Frequency preference was calculated as the mean of the frequency spectra at the frequency $\pm 0.01 \mathrm{~Hz}$ subtracted by the mean of 1.5 times the frequency \pm 0.01 . This metric includes not only the enhancement of rhythmic structure at the frequency in question but the suppression of rhythmic structure at frequencies between the fundamental frequency and the first harmonic. Paired data were analyzed using a Wilcoxon signed rank test with a Bonferroni correction for multiple comparisons where appropriate. Confidence intervals were determined from 5\% to $95 \%$ using bootstrapping with 1,000 iterations. 


\section{Results}

\section{Optogenetic in vitro model of slow cortical oscillation}

To probe the interaction dynamics between neocortical network activity and EF stimulation, we developed an experimental approach that enabled precise control of endogenous network dynamics in vitro, application of EF stimulation, and simultaneous recording of neuronal activity in all layers of cortical networks. To this end, we first determined if full-field optogenetic stimulation in absence of EF stimulation robustly evoked activity in the entire network and not just pyramidal cells in layer 5 that express ChR2 (Fig. 1A, left). We found that periodic stimulation with blue light $(460 \mathrm{~nm}$ optogenetic stimulation, $1 \mathrm{~Hz}, 500 \mathrm{~ms}$ of simulation followed by $500 \mathrm{~ms}$ without stimulation for each oscillation cycle) robustly evoked network activity (Fig. 1A, right). Importantly, individual SU in all cortical layers responded to optogenetic stimulation to varying degrees (Fig. 1B, activation ratio, longtailed distribution). The spread of activation ratios demonstrates that the SU in the stimulated slices did not all exclusively fire in lockstep (activation ratio of 1.0) with the stimulation but maintained their ability to spike in absence of stimulation (activation ratio of 0.5 if optogenetic stimulation had no effect). The SU maintained sparse firing (activation ratio, median \pm SEM: $0.907 \pm 0.003$ for all SU, $0.895 \pm 0.004$ for L 1-4, $0.917 \pm 0.004$ for L5\&6; FR: $1.30 \pm 0.038 \mathrm{~Hz}$ for all SU, $1.33 \pm 0.053 \mathrm{~Hz}$ for L1-4, $1.29 \pm 0.052 \mathrm{~Hz}$ for L5\&6; $n=3840,1536,2304 \mathrm{SU}^{*}$ trials, respectively; correlation: $\mathrm{r}=0.125, \mathrm{p}<10^{-3}$ for all SU; Fig 1B). Thus, the optogenetic stimulation of the slices did not cause unphysiological firing rates. Despite the cell-to-cell variability, the overall network activity in individual slices exhibited a prominent peak at $1 \mathrm{~Hz}$ in most trials $\left(1 \mathrm{~Hz}\right.$ peak: $1.05 \pm 0.027$ spikes $^{2} / \mathrm{Hz}$, $\mathrm{n}=703$ trials, trials sorted by power at $1 \mathrm{~Hz}$, Fig. 1C). These data demonstrate that our optogenetic stimulation paradigm provided a precisely tuned $1 \mathrm{~Hz}$ network oscillation but preserved in vivo-like sparseness and cell-to-cell variability of the firing patterns of individual neurons.

\section{Neocortex responds to weak EF with network resonance}

TACS provides a weak EF that we modeled with an applied EF generated by a voltagecontrolled current source. We simultaneously applied optogenetic stimulation to model endogenous network activity (Fig. 2A, Experimental set-up and waveforms for optogenetic and EF stimulation). We hypothesized that the response to stimulation is guided by network resonance near the frequency of the endogenous oscillation. Accordingly, EF stimulation of equal amplitude at the frequency (or its first harmonic) of the ongoing network oscillation would have a greater effect than stimulation with waveforms at nearby, yet mismatched frequencies. To test this hypothesis, we examined the network response to EF stimulation with two different amplitudes ( 1 and $2 \mathrm{mV} / \mathrm{mm}$ ) at stimulation frequencies matched to (1 $\mathrm{Hz})$, twice $(2 \mathrm{~Hz})$, and nearby $(0.8$ and $1.2 \mathrm{~Hz})$ the frequency of the endogenous network oscillation $(1 \mathrm{~Hz}$, induced by optogenetic stimulation). We first examined the raster plot of all $\mathrm{SU}$ for $1 \mathrm{~Hz}$ EF stimulation and determined that EF stimulation did not override the patterned network activity caused by optogenetic stimulation since oscillation cycles with and without EF closely resembled each other (Fig 2B, raster of all units for $1 \mathrm{~Hz}$ stimulation, $\mathrm{n}=3840 \mathrm{SU} *$ trials); peak activity occurred at the onset of the optogenetic stimulation during each oscillation cycle and low but detectable activity occurred in absence of 
optogenetic stimulation (second half of stimulation period). Therefore, stimulation with EF amplitudes similar to the ones estimated to occur in human neocortex during tACS [11, 27] failed to qualitatively alter the endogenous activity structure. In order to quantify the more subtle effects of EF stimulation on spontaneous cortical oscillations, we assessed the change in frequency structure of the overall network activity within individual slices by computing the change in frequency spectrum (Fig. 2C). In agreement with our hypothesis of frequencyspecific modulation, we found that these modulation profiles of oscillation structure exhibited distinct shapes for different stimulation frequencies $(0.8,1.0,1.2,2.0 \mathrm{~Hz})$. Independent of the stimulation frequency, the only clear peaks of enhancement were localized at or very close to $1 \mathrm{~Hz}$ (and its integer multiples). Therefore, our data reject the alternative hypothesis that EF stimulation enables the selective enhancement of network activity at the stimulation frequency. Rather, enhancement was strongly constrained by the endogenous oscillation, in particular in cases such as here where we induced a pronounced, in vivo-like slow oscillation by means of optogenetic stimulation. We quantified the modulation of the oscillatory activity by first determining the amount of enhancement at the EF stimulation frequency. For $1 \mathrm{mV} / \mathrm{mm}$ EF amplitude, we exclusively found an increase in frequency preference for EF stimulation frequencies of 1 and $2 \mathrm{~Hz}$. However, for increased stimulation amplitude ( $2 \mathrm{mV} / \mathrm{mm})$, the EF application significantly enhanced 1, 1.2, and 2 $\mathrm{Hz}$ oscillations respectively (Fig. 2D; confidence intervals and statistics Supplementary Table 1). These results show that cortex preferentially responded to EF stimulation at the frequency of endogenous oscillation and its first harmonic. We then examined the effect of the stimulation on the endogenous activity at $1 \mathrm{~Hz}$. Indeed, EF stimulation at the frequency of the network oscillation $(1 \mathrm{~Hz})$, its first harmonic $(2 \mathrm{~Hz})$, and below the network frequency $(0.8 \mathrm{~Hz})$ all enhanced the rhythmic activity caused by optogenetic stimulation at $1 \mathrm{~Hz}$ for low amplitude stimulation. EF stimulation amplitude to $2 \mathrm{mV} / \mathrm{mm}$ caused an increase of 1 $\mathrm{Hz}$ oscillation for all EF stimulation frequencies (Fig. 2E; Supplementary Table 2). Again, we found that perturbations at the frequency of the ongoing oscillation and the first harmonic preferentially enhance the ongoing oscillation. However, EF stimulation at the frequency below that of the ongoing oscillation also enhanced the oscillation. We therefore concluded that cortical networks respond to sine-wave EF stimulation, and likely by extension, tACS, with the properties of network resonance. EF stimulation predominantly enhanced the endogenously present oscillation and shifts in frequency of the endogenous were unattainable in these experiments. We next probed for outlasting effects by comparing the $10 \mathrm{~s}$ after EF enhancement to that of the $10 \mathrm{~s}$ preceding stimulation. We did not find any outlasting effect for any amplitude or frequency EF stimulation (Fig S1); this lack of outlasting effect is most likely due to the short duration of the EF stimulation and the randomization of the different stimulation frequency conditions in our study.

\section{EF stimulation increases neuronal activity levels}

Does EF stimulation only alter the temporal structure of network activity or does it also alter the net activity levels of individual neurons? To address this question, we next investigated how EF stimulation alters the overall activity levels of individual neurons by measuring firing rates (FRs) of SU. We examined modulation of FR during the UP state $(500 \mathrm{msec}$ activity caused by optogenetic stimulation), the DOWN state (500 $\mathrm{msec}$ of relative quiescence in absence of optogenetic stimulation), and the entire cycle while EF stimulation 
was applied compared to that of the preceding interval of optogenetic stimulation only (Fig. 3). Indeed application of most EF stimulations tested here increased FR during the UP state of the $1 \mathrm{~Hz}$ cycle (Fig 3A left, Supplementary Table 3). During the DOWN state, only EF stimulation that was matched to the frequency of the optogenetic stimulation $(1 \mathrm{~Hz} \mathrm{EF}$ stimulation) caused a FR decrease for both stimulation amplitudes (Fig 3A center, Supplementary Table 4). When we compared the total FR with EF stimulation to the FR without EF, we observed that $2 \mathrm{mV} / \mathrm{mm}$ EF enhanced FR for most stimulation frequencies (Fig. 3A right, Supplementary Table 5). However the total FR was not significantly increased for all low amplitude $(1 \mathrm{mV} / \mathrm{mm}) \mathrm{EF}$ stimulation frequencies except for mismatched frequency $(1.2 \mathrm{~Hz})$ stimulation. Thus in agreement with the findings on network oscillation structure, matching the stimulation frequency to the endogenous network frequency caused differential modulation of the network activity in contrast to non-matching stimulation frequencies.

We next assessed the temporal profile of the FR modulation by applied EF stimulation. In absence of EF stimulation, a pronounced increase in FR occurred at the onset of optogenetic stimulation followed by decay in FR with a relatively abrupt transition to low activity levels when the stimulus was turned off at $500 \mathrm{~ms}$ (black traces in Fig. 3B, left). Overall, this activity profile was maintained in presence of $\mathrm{EF}$ stimulation (blue and red traces in Fig $3 \mathrm{~B}$ left; Top: $1 \mathrm{~Hz}$ EF; Bottom: $2 \mathrm{~Hz} \mathrm{EF}$ ). We then calculated the percent change caused by application of EF and fitted a sine-wave to this time-profile of FR modulation (Fig 3B, right). We found that enhancement of neural activity by EF lagged behind the stimulus waveform for $1 \mathrm{~Hz}$ EF stimulation (goodness of fit: $\mathrm{R}^{2}=0.71$ for $1 \mathrm{mV} / \mathrm{mm}$ and 0.62 for 2 $\mathrm{mV} / \mathrm{mm} ; \varphi=-0.21$ for $1 \mathrm{mV} / \mathrm{mm}$ at $1 \mathrm{~Hz}$ and -0.14 for $2 \mathrm{mV} / \mathrm{mm}$ at $1 \mathrm{~Hz}$; Fig. 3B, top). Similarly, EF stimulation at $2 \mathrm{~Hz}$ caused a temporal modulation profile that followed a $2 \mathrm{~Hz}$ sine-wave ( $R^{2}=0.45$ for $1 \mathrm{mV} / \mathrm{mm}$ and 0.64 for $2 \mathrm{mV} / \mathrm{mm}$, Fig 3B bottom). Again we observed a phase delay between the EF stimulation and the change in $\mathrm{FR}(\varphi=-0.58$ for 1 $\mathrm{mV} / \mathrm{mm}$ at $1 \mathrm{~Hz}$ and -0.74 for $2 \mathrm{mV} / \mathrm{mm}$ at $2 \mathrm{~Hz}$; for results of 0.8 and $1.2 \mathrm{~Hz}$ EF see Fig S2). We then examined pairwise correlations between single units to determine if the increase in oscillatory structure was caused by an increase in similarity of firing patterns of individual neurons at a finer time-scale. Pairwise correlations on the $50 \mathrm{~ms}$ timescale were virtually unaltered (Supplementary Table 6). The increased oscillatory structure did thus not depend on increased synchronization at faster time-scales. Together, these results demonstrated an overall increase in FR for EF stimulation (with the exception of lowamplitude, frequency-matched stimulation) and a temporal modulation of activity levels that closely followed the applied EF waveform.

\section{Conditions that limit occurrence of network resonance}

Our finding of endogenous network dynamics constraining the effect of stimulation is likely most prevalent in the case of pronounced endogenous activity and low-amplitude periodic perturbations. To experimentally delineate the conditions in which the above demonstrated interaction dynamics apply, we performed additional control experiments. First, we conducted control experiments with $2 \mathrm{mV} / \mathrm{mm} \mathrm{EF}$ in the absence of optogenetic stimulation. In these experiments, the network activity without EF stimulation did not exhibit any peak in the frequency spectrum (Fig 4A). In contrast to the results presented above, the EF 
application enhanced the oscillatory structure at the frequency of the EF stimulation (median enhancement at stimulation frequency: 0.102 [0.076 0.132] spikes ${ }^{2} / \mathrm{Hz}$ for $0.8 \mathrm{~Hz} \mathrm{EF}, 0.067$ [0.032 0.101] spikes $^{2} / \mathrm{Hz}$ for $1.0 \mathrm{~Hz} \mathrm{EF}, 0.073$ [0.038 0.104] spikes $^{2} / \mathrm{Hz}$ for $1.2 \mathrm{~Hz} \mathrm{EF}$, and 0.059 of [0.043 0.071$]$ spikes $^{2} / \mathrm{Hz}$ for $2.0 \mathrm{~Hz} \mathrm{EF} ; \mathrm{p}<10^{-3}$ for each stimulation frequency; $\mathrm{n}$ $=144$ trials; Fig. 4B-C left). In further contrast to experiments with a strong oscillation evoked by the optogenetic stimulation, only EF stimulation of 1.0 and $2.0 \mathrm{~Hz}$ increased the frequency preference for $1.0 \mathrm{~Hz}$ oscillations (median enhancement at $1 \mathrm{~Hz}: 0.009$ [-0.015 0.016] spikes $2 / \mathrm{Hz}$ for $0.8 \mathrm{~Hz} \mathrm{EF}, 0.067$ [0.039 0.103] spikes $2 / \mathrm{Hz}$ for $1.0 \mathrm{~Hz} \mathrm{EF}, 0.015$ [-0.010 0.027] spikes $2 / \mathrm{Hz}$ for $1.2 \mathrm{~Hz} \mathrm{EF,} \mathrm{and} 0.055$ [0.034 0.075] spikes $2 / \mathrm{Hz}$ for $2.0 \mathrm{~Hz} \mathrm{EF}$; $\mathrm{p}=1, \mathrm{p}<10^{-3}, \mathrm{p}=1, \mathrm{p}<10^{-3}$ respectively; $\mathrm{n}=144$ trials; Fig. $4 \mathrm{C}$, right). Therefore, without an endogenous oscillation, EF stimulation increased oscillatory structure at all stimulation frequencies evaluated. In agreement with our overall hypothesis, these results suggest that the structure of the endogenous activity determines if and to what extent $\mathrm{EF}$ are constrained in their effect on network dynamics. Thus, we next investigated two other scenarios in which we reduced the strength of the endogenous oscillation modeled by optogenetic stimulation. We hypothesized that a reduction in endogenous rhythmic structure would enable even frequency-mismatched stimulation waveforms to enhance oscillations at the EF stimulation frequency.

First, we lowered the intensity of the optogenetic stimulation to $20 \mu \mathrm{W}$. With this lower intensity stimulation, the strength of the evoked oscillation was lower than that caused by the full intensity stimulation $\left(1.15\right.$ [1.12 1.24] spikes $^{2} / \mathrm{Hz}$ for low intensity optogenetic stimulation, 3.49 [3.35 3.64] spikes $^{2} / \mathrm{Hz}$ full intensity, $\mathrm{p}<10^{-3}$ Wilcoxon ranked sum test, $\mathrm{n}$ $=640$ and 703 trials respectively, Fig 5A). We then applied EF stimulation with $2 \mathrm{mV} / \mathrm{mm}$ amplitude. Similarly to the experiments with no optogenetic stimulation, EF stimulation was able to increase frequency preference at all stimulation frequencies evaluated (median enhancement for EF stimulation frequency: 0.068 [0.022 0.124] spikes $^{2} / \mathrm{Hz}$ for $0.8 \mathrm{~Hz} \mathrm{EF}$, 0.173 [0.097 0.260] spikes $^{2} / \mathrm{Hz}$ for $1.0 \mathrm{~Hz} \mathrm{EF}, 0.107$ [0.075 0.140] spikes $^{2} / \mathrm{Hz}$ for $1.2 \mathrm{~Hz}$ $\mathrm{EF}$, and 0.047 [0.016 0.083] spikes ${ }^{2} / \mathrm{Hz}$ for $2.0 \mathrm{~Hz} \mathrm{EF} ; \mathrm{p}<0.005$ for each stimulation frequency; $n=160$ trials; Fig. 5B-C left). However, the increase in oscillatory structure at the EF stimulation frequency trended to be larger for $1.0 \mathrm{~Hz}$ stimulation (confidence that response to $1 \mathrm{~Hz}$ stimulation was different: $\mathrm{p}=0.0898$ for $0.8 \mathrm{~Hz}$ stimulation, $\mathrm{p}=0.0595$ for $1.2 \mathrm{~Hz}$ stimulation and $\mathrm{p}=0.0029$ for $2 \mathrm{~Hz}$ stimulation, Wilcoxon ranked sum test with a Bonferroni correction for 3 comparisons). The frequency preference for the ongoing cortical oscillation was only enhanced for frequency matched $(1 \mathrm{~Hz})$ stimulation (median enhancement for optogenetic stimulation frequency: -0.052 [-0.125 -0.009] spikes ${ }^{2} / \mathrm{Hz}$ for $0.8 \mathrm{~Hz} \mathrm{EF}, 0.173$ [0.101 0.261] spikes $^{2} / \mathrm{Hz}$ for $1.0 \mathrm{~Hz} \mathrm{EF},-0.048$ [-0.110 -0.009] spikes $^{2} / \mathrm{Hz}$ for $1.2 \mathrm{~Hz} \mathrm{EF}$, and 0.039 [-0.015 0.080] spikes $^{2} / \mathrm{Hz}$ for $2.0 \mathrm{~Hz} \mathrm{EF} ; \mathrm{p}=0.026, \mathrm{p}$ $<10^{-3}, \mathrm{p}=0.167$, and $\mathrm{p}=0.383$ respectively; $\mathrm{n}=160$ trials; Fig. $5 \mathrm{C}$ right). Thus, with a less pronounced endogenous oscillation, EF stimulation provided the best enhancement when stimulating at the frequency of the ongoing oscillation. However mismatches stimulation also enhanced frequency preference for the EF stimulation frequency.

Second, we reduced the strength of the ongoing oscillation by using a optogenetic stimulation waveform with a broader spectral peak around $1 \mathrm{~Hz}$. Specifically, we varied the length of each "UP" and "DOWN" state by randomly drawing 20 durations from a normal 
distribution around $500 \mathrm{~ms}$ (standard deviation $50 \mathrm{~ms}$ ). We then compared the multiunit frequency response to optogenetic stimulation of the experiments shown in Figs. 1-3 to the response to the variable duration optogenetic stimulation. The frequency structure of the stimulation waveform still exhibited a peak frequency of $1 \mathrm{~Hz}$. However, the frequency content was spread over a broader band (Fig. 6A). Due to this change in the optogenetic stimulation, the evoked cortical oscillation was also altered but still exhibited a peak frequency at $1.0 \mathrm{~Hz}$ (Fig. 6B). However, the cortical response at 0.8 and $1.2 \mathrm{~Hz}$ was relatively enhanced when normalized by the peak $(1 \mathrm{~Hz})$ amplitude (Supplementary Table 7 , Fig. $6 \mathrm{~B}$ right). We then applied $2 \mathrm{mV} / \mathrm{mm}$ EF stimulation to slices activated with this optogenetic stimulation paradigm. Similarly to the results in (Fig 2C), EF stimulation increased the frequency preference for $1.0 \mathrm{~Hz}$ using 1.0, 1.2 and $2.0 \mathrm{~Hz}$ stimulation, however EF stimulation at $0.8 \mathrm{~Hz}$ did not increase the frequency preference for $1.0 \mathrm{~Hz}$ (median enhancement at optogenetic stimulation frequency, $1 \mathrm{~Hz}$ : 0.003 [-0.044 0.048] spikes $^{2} / \mathrm{Hz}$ for $0.8 \mathrm{~Hz} \mathrm{EF}, 0.199$ [0.127 0.289] spikes $^{2} / \mathrm{Hz}$ for $1.0 \mathrm{~Hz} \mathrm{EF}, 0.063$ [-0.021 0.134] spikes $^{2} / \mathrm{Hz}$ for $1.2 \mathrm{~Hz} \mathrm{EF}$, and 0.148 [0.053 0.222] spikes $^{2} / \mathrm{Hz}$ for $2.0 \mathrm{~Hz} \mathrm{EF} ; \mathrm{p}=1, \mathrm{p}$ $<10^{-3}, \mathrm{p}=0.014$ and $\mathrm{p}=0.001$ respectively; $\mathrm{n}=176$ trials; Fig $6 \mathrm{C} \& 6 \mathrm{D}$ left). With increased frequency structure at 0.8 and $1.2 \mathrm{~Hz}$ in the optogenetic stimulation, the $\mathrm{EF}$ stimulation was able to significantly enhance frequency preference for the applied EF frequency (median enhancement at EF stimulation frequency: .446 [0.348 0.532$]$ spikes $^{2} / \mathrm{Hz}$ for $0.8 \mathrm{~Hz} \mathrm{EF}, 0.198$ [0.127 0.275] spikes $^{2} / \mathrm{Hz}$ for $1.0 \mathrm{~Hz} \mathrm{EF}, 0.092$ [0.038 0.161] spikes $^{2} / \mathrm{Hz}$ for $1.2 \mathrm{~Hz} \mathrm{EF}$, and 0.104 [0.040 0.157] spikes $^{2} / \mathrm{Hz}$ for $2.0 \mathrm{~Hz} \mathrm{EF}$; $<<10^{-3}$ for all groups; $n=176$ trials; Fig $6 \mathrm{D}$ right). Therefore, the ongoing variable oscillation also shaped the network response to EF stimulation.

Finally, we asked if increasing the strength of the EF stimulation would enable the EF stimulation to override the endogenous network dynamics. To answer this question, we performed experiments with $8 \mathrm{mV} / \mathrm{mm}$ field strength. At such high amplitude, the EF stimulation was indeed able to increase frequency preference for the EF frequency despite the ongoing oscillation at $1 \mathrm{~Hz}$ (median enhancement at EF stimulation frequency: 0.382 [0.258 0.560] spikes $2 / \mathrm{Hz}$ for $0.8 \mathrm{~Hz} \mathrm{EF}, 0.471$ [0.221 0.661] spikes $^{2} / \mathrm{Hz}$ for $1.0 \mathrm{~Hz} \mathrm{EF}$, 0.319 [0.173 0.441] spikes ${ }^{2} / \mathrm{Hz}$ for $1.2 \mathrm{~Hz} \mathrm{EF}$, and of 0.185 [0.130 0.290] spikes $^{2} / \mathrm{Hz}$ for 2.0 $\mathrm{Hz}$ EF; $\mathrm{p}<10^{-3} ; \mathrm{n}=208$ trials; Fig 7A-B left). Only EF stimulation at $1.0 \mathrm{~Hz}$ increased the frequency preference for $1.0 \mathrm{~Hz}$ while $\mathrm{EF}$ stimulation at $0.8 \mathrm{~Hz}$ suppressed the frequency preference for $1.0 \mathrm{~Hz}$ (median enhancement at optogenetic stimulation frequency: -0.142 [-0.209-0.049] spikes $^{2} / \mathrm{Hz}$ for $0.8 \mathrm{~Hz} \mathrm{EF}, 0.483$ [0.223 0.661] spikes $^{2} / \mathrm{Hz}$ for $1.0 \mathrm{~Hz} \mathrm{EF}$, 0.0444 [0.000 0.126] spikes $2 / \mathrm{Hz}$ for $1.2 \mathrm{~Hz} \mathrm{EF}$, and 0.100 [-0.018 0.245] spikes $^{2} / \mathrm{Hz}$ for 2.0 $\mathrm{Hz}$ EF; $\mathrm{p}=0.017, \mathrm{p}<10^{-3}, \mathrm{p}=0.915$ and $\mathrm{p}=0.054$ respectively; $\mathrm{n}=208$ trials; Fig 7B right). From these data, we concluded that indeed a comparatively strong EF may override the response-dependence of EF stimulation on the ongoing cortical oscillation.

\section{Discussion}

Targeted modulation of cortical oscillations with non-invasive brain stimulation has the potential to enable significant progress (1) in the study of the causal role of oscillations in cognition and behavior and (2) in the development of novel treatments for neurological and psychiatric disorders associated with deficits in the macroscopic cortical activity patterns. 
However, in absence of a direct demonstration of the underlying mechanism of action by which such stimulation interacts with ongoing network dynamics, this progress will be severely hampered.

Application of weak EFs by transcranial current stimulation represents one promising approach to noninvasive brain stimulation [30-32]. The presence of endogenous activity is crucial for EFs to have an effect on network dynamics since the depolarization caused by the $\mathrm{EF}$ is too small to activate neurons at rest [14, 17, 33]. In essence, neurons need to be close to firing threshold for the resulting sub-millivolt perturbation of the membrane voltage to be an effective modulator of endogenous network activity. Previous in vitro studies have shown enhancing effects of sine-wave EF stimulation on ongoing network activity [13-15].

However, the direct assessment of the interaction dynamics has been limited to observational descriptions of the effects of weak EF due to limited control of spontaneous or pharmacologically induced oscillations. Here, we used optogenetic tools to directly assess these interaction dynamics by exercising precise temporal control of both the endogenous activity (induced by optogenetic stimulation) and the EF stimulation. We established that sine-wave EF patterns with very low amplitude can indeed enhance network dynamics even in presence of very pronounced, rhythmic neocortical network activity that resembles in vivo activity patterns. In agreement with predictions from previous experimental $[15,34]$ and computational work [17], we show that the choice of stimulation frequency relative to the endogenous oscillation frequency plays a key role in determining the effect of stimulation. Specifically, we found that a pronounced endogenous oscillation precluded the generation of activity patterns at other (nearby) frequencies and that the enhancement of the endogenous oscillation depended on the EF stimulation frequency. We then tested the apparent constraint endogenous network dynamics impose on modulatory effects of stimulation; indeed, with no endogenous oscillation present or with application of higher amplitude EF stimulation, oscillations at the EF stimulation frequency could be enhanced. We also reduced the strength of the endogenous oscillation (by reduced optogenetic stimulation) and found that these dynamics still constrained the effects of stimulation at trend level. Finally, with a temporally varied endogenous oscillation, we again found that mismatched stimulation could increase the endogenous oscillation but also increased oscillatory structure at the EF stimulation frequency.

The field has yet to converge on whether application of EF increases the net average FR of networks. In our hands, the average FR over $10 \mathrm{~s}$ was increased for most of the $2 \mathrm{mV} / \mathrm{mm}$ EF stimulation frequencies. This result agrees with the previously reported increase in multiunit activity evoked by field strengths as low as $1 \mathrm{mV} / \mathrm{mm}$ in [14]. However other studies have observed that while EF stimulation increased FR during the positive going phase of the stimulation, during the negative-going phase of the stimulation the FR was decreased, thus resulting in no net change [15]. This distinction may be important for future studies aimed at determining if tACS acts by synchronizing neural oscillators or evoking more activity as a function of the temporal structure of the stimulation waveform.

Our findings demonstrate that the state of the targeted networks plays an important role in determining the response to EF stimulation. Such state-dependent response of neuronal circuits is well known for sensory input that reaches sensory areas in cortex. For example, 
the neuronal representation of visual [35] and auditory [36] inputs depends on overall brain state. Our results suggest that this "gating" by brain state (defined by the presence of specific network-level activity patterns) also applies to external stimuli directly provided to neuronal networks by brain stimulation. Matching the brain stimulation to the specific endogenous network dynamics of an individual at a given time-point may therefore be crucial for effectiveness and thus an appealing opportunity for individualized treatment. Indeed, in human motor cortex, the presence of a pronounced beta oscillation was found to determine whether beta $(20 \mathrm{~Hz})$ or theta $(5 \mathrm{~Hz})$ frequency tACS best facilitated motorevoked potentials by transcranial magnetic stimulation [34]. Here we have performed EF stimulation in the presence and absence of a cortical oscillation and likewise observed different cortical response dependent on the cortical oscillation. In contrast, we used EF stimulation frequencies within the same frequency band as the ongoing cortical oscillation since oscillations in different frequency bands are likely generated by different mechanisms and presently cannot be simultaneously modeled in a slice preparation. Importantly, a similar phenomenon has also been reported for alpha oscillations where the effect of tACS on macroscopic network dynamics depended on the strength of the ongoing oscillation [37]. However, in this case, the presence of strong alpha oscillations precluded further enhancement by frequency-matched tACS. Yet, enhancement was successful for the case of weak endogenous alpha oscillations in the same participants. This difference in the effect of frequency-matched stimulation can have several causes, including the presence of ceiling effects. Taken together with the results presented here, the effect of EF stimulation may vary from enhancement at any frequency, to enhancement only of the endogenous oscillation, to no enhancement at the stimulation frequency, depending on the strength of the ongoing cortical oscillation.

Importantly, further studies will be needed to fully elucidate the mechanism of action of tACS for other cortical oscillations with different frequency structure such as the gamma oscillation $(>30 \mathrm{~Hz})$. Since cortical oscillations at different frequencies are likely generated by distinct single cell and network mechanisms, it is as of now unclear if the work presented here will generalize to other cortical oscillations. For example, the gamma oscillation likely arises from the dynamic interaction of pyramidal cells and fast-spiking inhibitory interneurons that exhibit their own resonance profile [38]. Nevertheless, the overall approach taken in this study will likely be equally elucidating when applied to the study of tACS that targets other frequency bands and therefore lays the groundwork for a comprehensive understanding of tACS and its interaction with cortical network dynamics [39].

Our study focused on unraveling the interaction dynamics between periodic, weak EF and oscillatory network activity. Understanding tACS will require the study of other important aspects of stimulation delivery. In particular, we used a model system of tACS where we directly applied calibrated EF to cortical slices in vitro and recorded microscopic network dynamics measured by SU activity. Thus, our study does not provide any insight into the spatial patterning of the stimulation (i.e. EF distribution) as a result of the current application to the scalp in human tACS. Only the intersection of the mechanisms of temporal targeting (demonstrated here), spatial targeting [40], and functional targeting [34] 
will provide the tools for developing highly specific stimulation paradigms. We also cannot, with only one slice, examine the mechanisms of applying anti-phase tACS to different brain regions. This type of stimulation has shown interesting behavioral results [8, 41], but cannot be probed with the methods used here. Likely, distinct mechanisms such as switching between multiple stable states may mediate such effects on coherence [42]. Nevertheless, our in vitro approach provides essential advantages that can help build an important bridge between computational studies (that inherently lack biological plausibility) and in vivo studies in animals and humans that often do not provide the level of experimental access and control required to elucidate the interaction dynamics. Using optogenetic stimulation of layer 5 pyramidal cells causes naturalistic slow rhythmic activity in vivo; here we adapted this approach for in vitro stimulation. We found such stimulation to cause a clear network rhythm although individual cells continued to exhibit low, naturalistic firing rates in presence of stimulation. Given the known role of layer 5 pyramidal cell to greatly contribute to the genesis of slow (cortical) rhythms, our approach provided the opportunity to maintain in vivo like activity patterns in vitro.

Together, these results provide a mechanism of action of tACS at the network level and point towards next-generation tACS that provides individualized, state-dependent feedback tACS for the enhancement of cortical oscillations [43]. The most important next step towards this goal will be the successful recording of brain activity during stimulation as recently demonstrated for EEG combined with tACS [44].

\section{Supplementary Material}

Refer to Web version on PubMed Central for supplementary material.

\section{Acknowledgments}

The authors thank the Fröhlich lab and the anonymous reviewers for their valuable input. Research reported in this publication was supported in part by the National Institute of Mental Health of the National Institutes of Health under Award Number R01MH101547. The content is solely the responsibility of the authors and does not necessarily represent the official views of the National Institutes of Health. AAF was funded by the Summer Undergraduate Research Fellowship from the Office of Undergraduate Research at University of North Carolina at Chapel Hill.

\section{References}

1. Buzsáki, G. Rhythms of the brain2006. Vol. xiv. Oxford University Press; Oxford: New York: p. 448

2. Wang XJ. Neurophysiological and computational principles of cortical rhythms in cognition. Physiological reviews. 2010; 90(3):1195-268. [PubMed: 20664082]

3. Uhlhaas PJ, Singer W. Neuronal dynamics and neuropsychiatric disorders: toward a translational paradigm for dysfunctional large-scale networks. Neuron. 2012; 75(6):963-80. [PubMed: 22998866]

4. Romei V, Gross J, Thut G. On the role of prestimulus alpha rhythms over occipito-parietal areas in visual input regulation: correlation or causation? The Journal of neuroscience : the official journal of the Society for Neuroscience. 2010; 30(25):8692-7. [PubMed: 20573914]

5. Sauseng P, et al. Brain oscillatory substrates of visual short-term memory capacity. Current biology : CB. 2009; 19(21):1846-52. [PubMed: 19913428]

6. Thut G, et al. Rhythmic TMS causes local entrainment of natural oscillatory signatures. Current biology : CB. 2011; 21(14):1176-85. [PubMed: 21723129] 
7. Herrmann CS, et al. Transcranial alternating current stimulation: a review of the underlying mechanisms and modulation of cognitive processes. Frontiers in human neuroscience. 2013; 7:279. [PubMed: 23785325]

8. Polania R, et al. The importance of timing in segregated theta phase-coupling for cognitive performance. Current biology : CB. 2012; 22(14):1314-8. [PubMed: 22683259]

9. Santarnecchi E, et al. Frequency-dependent enhancement of fluid intelligence induced by transcranial oscillatory potentials. Current biology : CB. 2013; 23(15):1449-53. [PubMed: 23891115]

10. Kirov R, et al. Slow oscillation electrical brain stimulation during waking promotes EEG theta activity and memory encoding. Proceedings of the National Academy of Sciences of the United States of America. 2009; 106(36):15460-5. [PubMed: 19706399]

11. Marshall L, et al. Boosting slow oscillations during sleep potentiates memory. Nature. 2006; 444(7119):610-3. [PubMed: 17086200]

12. Binder $S$, et al. Transcranial slow oscillation stimulation during NREM sleep enhances acquisition of the radial maze task and modulates cortical network activity in rats. Frontiers in behavioral neuroscience. 2014; 7:220. [PubMed: 24409131]

13. Deans JK, Powell AD, Jefferys JG. Sensitivity of coherent oscillations in rat hippocampus to AC electric fields. The Journal of physiology. 2007; 583(Pt 2):555-65. [PubMed: 17599962]

14. Frohlich F, McCormick DA. Endogenous electric fields may guide neocortical network activity. Neuron. 2010; 67(1):129-43. [PubMed: 20624597]

15. Reato D, et al. Low-intensity electrical stimulation affects network dynamics by modulating population rate and spike timing. The Journal of neuroscience : the official journal of the Society for Neuroscience. 2010; 30(45):15067-79. [PubMed: 21068312]

16. Ozen $\mathrm{S}$, et al. Transcranial electric stimulation entrains cortical neuronal populations in rats. The Journal of neuroscience : the official journal of the Society for Neuroscience. 2010; 30(34):1147685. [PubMed: 20739569]

17. Ali MM, Sellers KK, Frohlich F. Transcranial alternating current stimulation modulates large-scale cortical network activity by network resonance. The Journal of neuroscience : the official journal of the Society for Neuroscience. 2013; 33(27):11262-75. [PubMed: 23825429]

18. Neuling T, Rach S, Herrmann CS. Orchestrating neuronal networks: sustained after effects of transcranial alternating current stimulation depend upon brain states. Frontiers in human neuroscience. 2013; 7:161. [PubMed: 23641206]

19. Hutcheon B, Yarom Y. Resonance, oscillation and the intrinsic frequency preferences of neurons. Trends in neurosciences. 2000; 23(5):216-22. [PubMed: 10782127]

20. Beltramo R, et al. Layer-specific excitatory circuits differentially control recurrent network dynamics in the neocortex. Nature neuroscience. 2013; 16(2):227-34.

21. Arenkiel BR, et al. In vivo light-induced activation of neural circuitry in transgenic mice expressing channelrhodopsin-2. Neuron. 2007; 54(2):205-18. [PubMed: 17442243]

22. Chauvette $S$, Volgushev M, Timofeev I. Origin of active states in local neocortical networks during slow sleep oscillation. Cerebral cortex. 2010; 20(11):2660-74. [PubMed: 20200108]

23. Schmidt SL, et al. Differential effects of cholinergic and noradrenergic neuromodulation on spontaneous cortical network dynamics. Neuropharmacology. 2013; 72:259-73. [PubMed: 23643755]

24. Sanchez-Vives MV, McCormick DA. Cellular and network mechanisms of rhythmic recurrent activity in neocortex. Nature neuroscience. 2000; 3(10):1027-34.

25. Tranchina D, Nicholson C. A model for the polarization of neurons by extrinsically applied electric fields. Biophysical journal. 1986; 50(6):1139-56. [PubMed: 3801574]

26. Bikson M, et al. Effects of uniform extracellular DC electric fields on excitability in rat hippocampal slices in vitro. The Journal of physiology. 2004; 557(Pt 1):175-90. [PubMed: 14978199]

27. Datta A, et al. Gyri-precise head model of transcranial direct current stimulation: improved spatial focality using a ring electrode versus conventional rectangular pad. Brain stimulation. 2009; 2(4): 201-7. 207, e1. [PubMed: 20648973] 
28. Fee MS, Mitra PP, Kleinfeld D. Automatic sorting of multiple unit neuronal signals in the presence of anisotropic and non-Gaussian variability. Journal of neuroscience methods. 1996; 69(2):17588. [PubMed: 8946321]

29. Hill DN, Mehta SB, Kleinfeld D. Quality metrics to accompany spike sorting of extracellular signals. The Journal of neuroscience : the official journal of the Society for Neuroscience. 2011; 31(24):8699-705. [PubMed: 21677152]

30. Brunoni AR, et al. Understanding tDCS effects in schizophrenia: a systematic review of clinical data and an integrated computation modeling analysis. Expert review of medical devices. 2014; 11(4):383-94. [PubMed: 24754366]

31. Reato D, et al. Effects of weak transcranial alternating current stimulation on brain activity-a review of known mechanisms from animal studies. Frontiers in human neuroscience. 2013; 7:687. [PubMed: 24167483]

32. Kuo MF, Paulus W, Nitsche MA. Therapeutic effects of non-invasive brain stimulation with direct currents (tDCS) in neuropsychiatric diseases. NeuroImage. 2014; 85(Pt 3):948-60. [PubMed: 23747962]

33. Radman T, et al. Role of cortical cell type and morphology in subthreshold and suprathreshold uniform electric field stimulation in vitro. Brain stimulation. 2009; 2(4):215-28. 228, e1-3. [PubMed: 20161507]

34. Feurra M, et al. State-dependent effects of transcranial oscillatory currents on the motor system: what you think matters. The Journal of neuroscience : the official journal of the Society for Neuroscience. 2013; 33(44):17483-9. [PubMed: 24174681]

35. Niell CM, Stryker MP. Modulation of visual responses by behavioral state in mouse visual cortex. Neuron. 2010; 65(4):472-9. [PubMed: 20188652]

36. Curto C, et al. A simple model of cortical dynamics explains variability and state dependence of sensory responses in urethane-anesthetized auditory cortex. The Journal of neuroscience : the official journal of the Society for Neuroscience. 2009; 29(34):10600-12. [PubMed: 19710313]

37. Neuling T, et al. Finite-Element Model Predicts Current Density Distribution for Clinical Applications of tDCS and tACS. Frontiers in psychiatry. 2012; 3:83. [PubMed: 23015792]

38. Cardin JA, et al. Driving fast-spiking cells induces gamma rhythm and controls sensory responses. Nature. 2009; 459(7247):663-7. [PubMed: 19396156]

39. Frohlich F, Schmidt SL. Rational design of transcranial current stimulation (TCS) through mechanistic insights into cortical network dynamics. Frontiers in human neuroscience. 2013; 7:804. [PubMed: 24324427]

40. Kuo HI, et al. Comparing cortical plasticity induced by conventional and high-definition $4 \times 1$ ring tDCS: a neurophysiological study. Brain stimulation. 2013; 6(4):644-8. [PubMed: 23149292]

41. Struber D, et al. Antiphasic $40 \mathrm{~Hz}$ oscillatory current stimulation affects bistable motion perception. Brain topography. 2014; 27(1):158-71. [PubMed: 23709044]

42. Kutchko KM, Frohlich F. Emergence of metastable state dynamics in interconnected cortical networks with propagation delays. PLoS computational biology. 2013; 9(10):e1003304. [PubMed: 24204238]

43. Boyle MR, Frohlich F. EEG Feedback-Controlled Transcranial Alternating Current Stimulation. 2013 6th International Ieee/Embs Conference on Neural Engineering (Ner). 2013:140-143.

44. Helfrich RF, et al. Entrainment of brain oscillations by transcranial alternating current stimulation. Current biology : CB. 2014; 24(3):333-9. [PubMed: 24461998] 


\section{Highlights}

Endogenous oscillations constrain neuromodulation by weak electric fields.

Weak electric fields enhance neocortical oscillations by resonance.

Frequency-matched tACS is likely most effective for enhancement of cortical rhythms. 
A

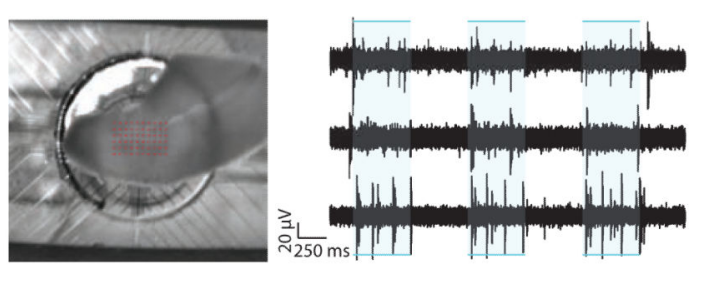

B

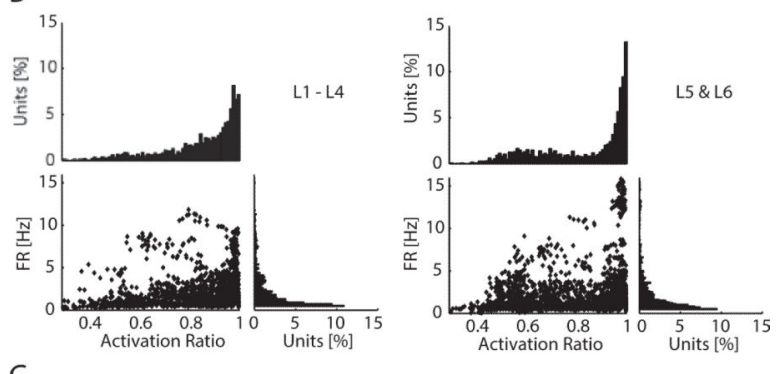

C

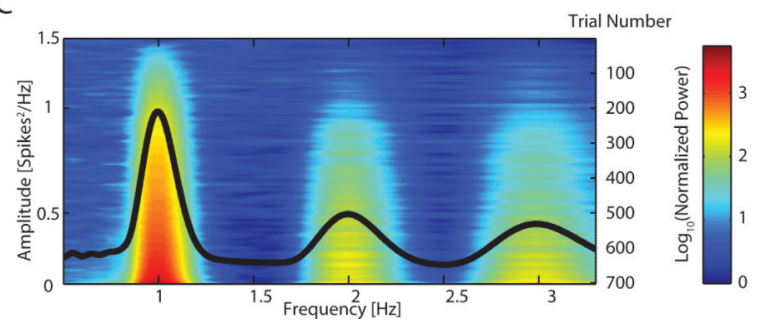

Figure 1. Optogenetic Stimulation Activates Cortical Networks in Vitro

(A) Left: Coronal slice positioned on multielectrode array with $100 \mu \mathrm{m}$ spacing between electrodes. Right: Modulation of action potential firing by optogenetic stimulation ( $2 \mathrm{~s}$ trace of multiunit activity for three sample electrodes) (B) FR versus Activation Ratio for all SU in layers 1 through 4 (left) and layers 5 and 6 (right). (C) Frequency response to $10 \mathrm{~s}$ of optogenetic stimulation with mean response overlaid (black). Spontaneous activity was modulated by optogenetic stimulation and exhibited pronounced $1 \mathrm{~Hz}$ oscillation. 

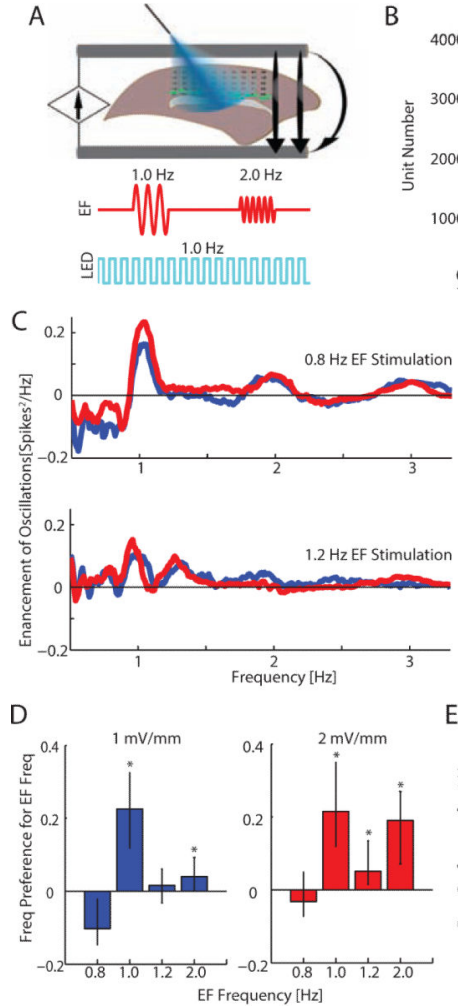
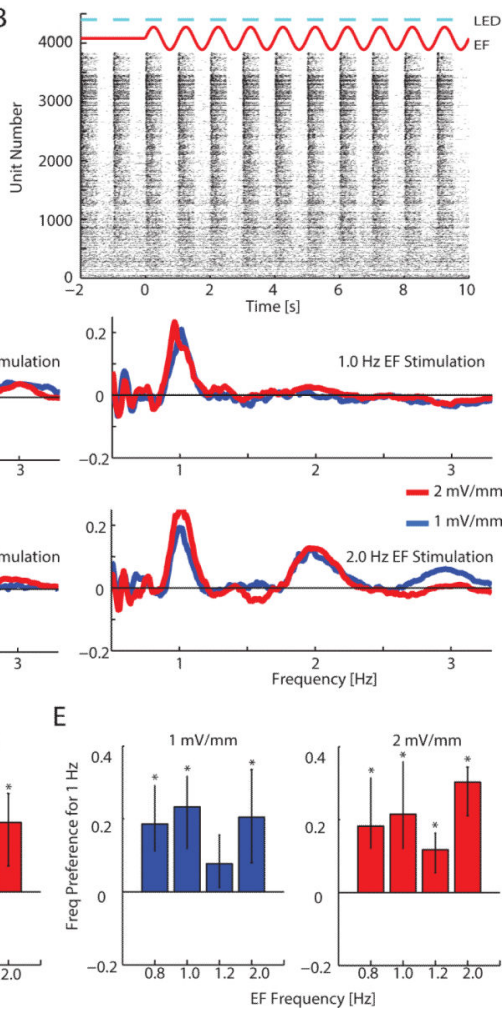

Figure 2. Interaction Dynamics between Endogenous Oscillation and EF Stimulation (A) Top: Optogenetic stimulation (cyan) is applied to L5 pyramidal neurons (green) while $\mathrm{EF}$ is applied between two $\mathrm{AgCl}$ wires (gray, example field lines in black) with the positive terminal nearest layer 1 of visual cortex. Bottom: Example trials (shortened to $3 \mathrm{~s}$ for visualization). (B) Raster plot for all SU for each trial with $1 \mathrm{~Hz}$ EF stimulation. (C) Difference spectra between activity during EF stimulation and the preceding 10s for all EF stimulation frequencies and amplitudes. (D) Frequency preference for the stimulation frequency. (E) Frequency preference for $1 \mathrm{~Hz}$. Error bars indicate 5 to $95 \%$ confidence interval. $* \mathrm{p}<0.05$. 

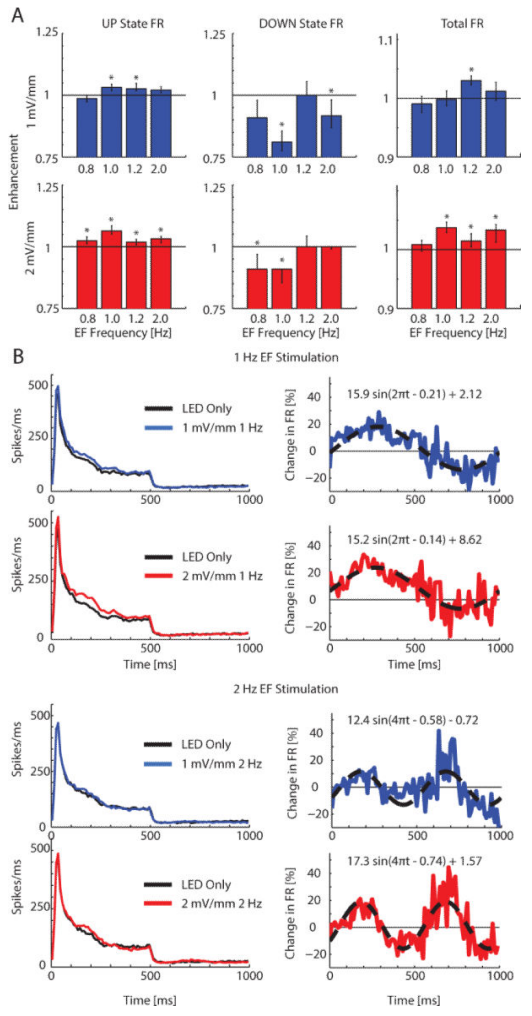

Figure 3. Firing Rate Modulation by EF Stimulation

(A) Left: Enhancement of FR during UP states. FR was enhanced by most EF stimulation amplitudes and frequencies during the UP state. Center: Enhancement of FR during the DOWN state. FR was significantly decreased for $1 \mathrm{~Hz}$ EF stimulation across stimulation amplitudes. Right: Total enhancement of FR by EF. In most cases application of $2 \mathrm{mV} / \mathrm{mm}$ EF increased total FR while only $1.2 \mathrm{~Hz}$ stimulation increased FR for $1 \mathrm{mV} / \mathrm{mm} \mathrm{EF}$. SU with an activation ratio of $<1$ (at least one spike during the DOWN state) shown. (B) Left: Spiking activity as a single cycle of the applied EF frequency for 1 and $2 \mathrm{~Hz}$ EF stimulation (color) with the averaged preceding $10 \mathrm{~s}$ of oscillatory activity (black). Right: Percent change by application of EF (color) and fitted sine-waves (black, dashed). Phase delay (shown in radians) between the applied EF signal and resulting change in activity was observed for both conditions. Error bars indicate 5 to $95 \%$ confidence interval. $* \mathrm{p}<0.05$. 

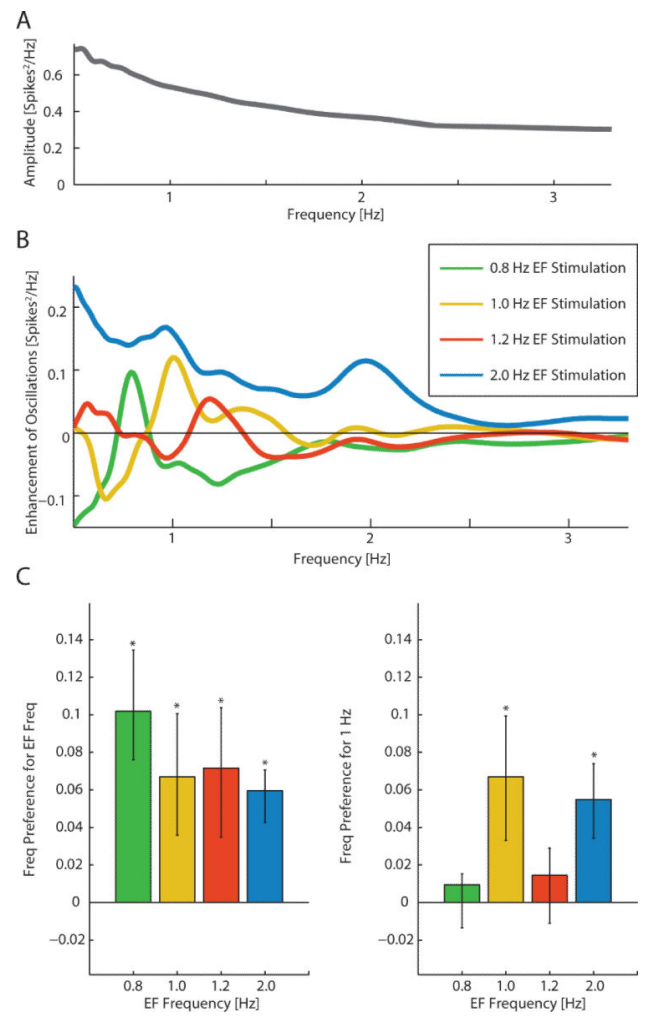

Figure 4. Modulation of Frequency Structure in Absence of an Endogenous Oscillation (A) Frequency spectrum of spontaneous activity in cortical slices with neither optogenetic stimulation nor EF stimulation. (B) Enhancement of frequency structure by EF stimulation only. (C) Frequency preference for the EF stimulation frequency (left). Frequency preference for $1 \mathrm{~Hz}$ oscillations (right). In absence of strong cortical oscillations, all frequencies were enhanced and only 1 and $2 \mathrm{~Hz}$ EF stimulation enhanced preference for 1 $\mathrm{Hz}$ oscillations. Error bars indicate 5 to $95 \%$ confidence interval. $* \mathrm{p}<0.05$. 

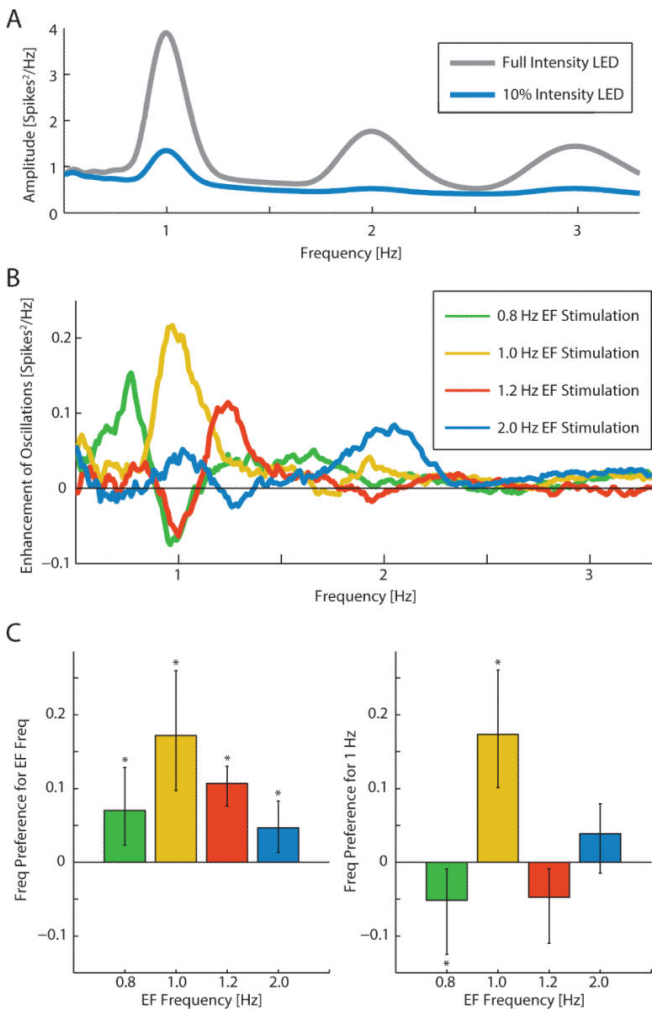

Figure 5. EF Stimulation of Networks Exhibiting Weak Cortical Oscillations

(A) Spectra of the response to full intensity optogenetic simulation (gray) and 10\% intensity optogenetic stimulation (blue). (B) Enhancement of cortical oscillations by EF stimulation.

(C) Change in frequency preference for the EF stimulation frequency (left) and for the $1 \mathrm{~Hz}$ oscillation (right). Error bars indicate 5 to $95 \%$ confidence interval. $* \mathrm{p}<0.05$. 

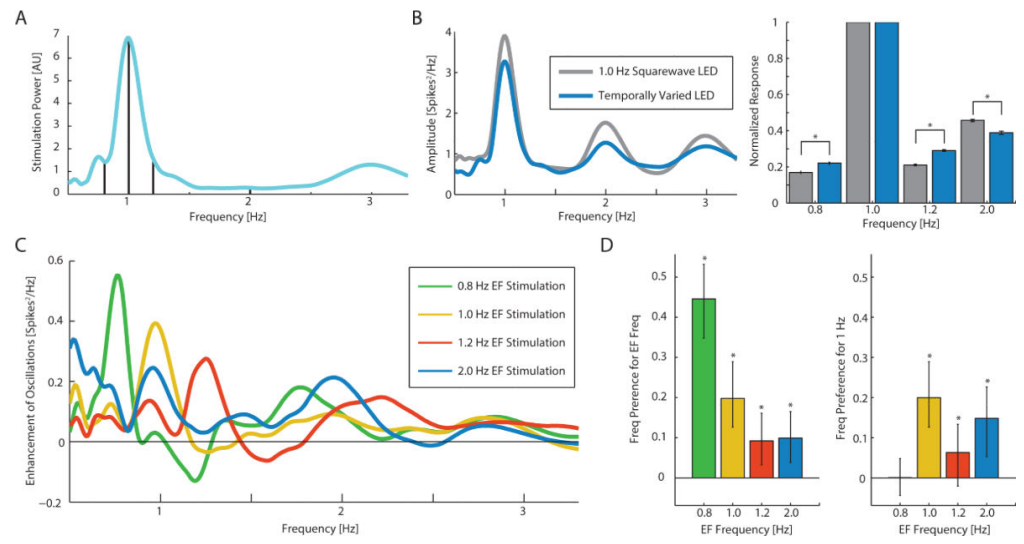

Figure 6. EF Stimulation of Networks Exhibiting Cortical Oscillations with Variable UP and DOWN state duration

(A) Spectrum of the $10 \mathrm{~s}$ optogenetic stimulation waveform (cyan). EF stimulation frequencies are indicated in black. (B) Left: Spectra of the response to $1 \mathrm{~Hz}$ square-wave optogenetic stimulation (gray) and response to temporally variant optogenetic stimulation (blue). Right: Response optogenetic stimulation, normalized by the oscillation amplitude at $1 \mathrm{~Hz}$. (C) Spectra of the enhancement of oscillations by EF stimulation for 0.8 (green), 1.0 (yellow), 1.2 (red), 2.0 (blue) Hz stimulation. (D) Left: Frequency preference for oscillations at the frequency of the EF stimulation. Right: Frequency preference for $1 \mathrm{~Hz}$ oscillations. Error bars indicate 5 to $95 \%$ confidence interval. $* \mathrm{p}<0.05$. 

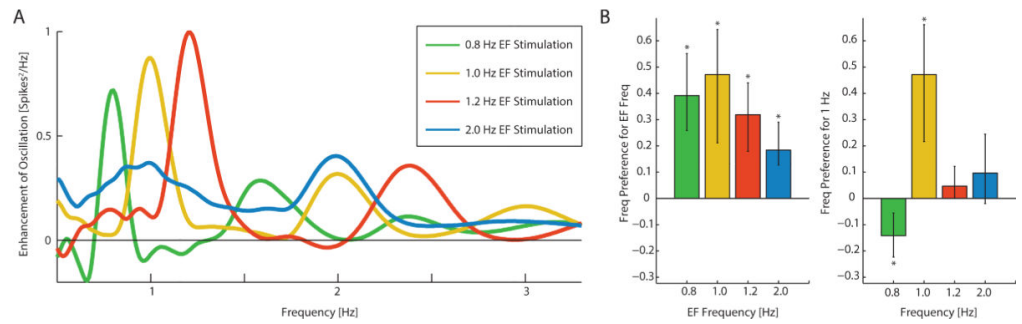

Figure 7. High Amplitude EF Stimulation

(A) Difference spectra between $10 \mathrm{~s}$ of application of $8 \mathrm{mV} / \mathrm{mm}$ EF stimulation and the preceding $10 \mathrm{~s}$. (B) Left: Frequency preference for the EF stimulation frequency. Right:

Frequency preference for $1 \mathrm{~Hz}$ stimulation. Error bars indicate 5 to $95 \%$ confidence interval. $* \mathrm{p}<0.05$ 